CERN-TH/96-145

hep-th/9606031

\title{
The string spectrum on the horizon of a non-extremal black hole
}

\author{
J.G. Russo \\ Theory Division, CERN \\ CH-1211 Geneva 23, Switzerland
}

\begin{abstract}
We investigate the conformal string $\sigma$-model corresponding to a general fivedimensional non-extremal black hole solution. In the horizon region the theory reduces to an exactly solvable conformal field theory. We determine the modular invariant spectrum of physical string states, which expresses the Rindler momentum operator in terms of three charges and string oscillators. For black holes with winding and Kaluza-Klein charges, we find that states made with only right-moving excitations have ADM mass equal to the black hole ADM mass, and thus they can be used as sources of the gravitational field. A discussion on statistical entropy is included.
\end{abstract}

June 1996 


\section{Introduction}

Recently two complementary approaches have shed light on the statistical origin of the entropy of extremal black holes in string theory. One is based on the D-brane representation of string solitons with RR charge (see e.g. [1,2]). This permits the counting of all BPS quantum states in the weakly coupled theory with given charges. This number is a topological quantity and it is unchanged in extrapolating from weak coupling to strong coupling where the black hole appears; thus it represents the degeneracy of the ground state of these extremal black holes. This method has provided a number of concrete results, although the extension beyond extremality (for black holes with Schwarzschild radii much larger than the compactification scale) appears to be problematic. The other approach, pursued for instance in refs. [3 [12], is based on the idea of interpreting the statistical entropy of black holes with NS charges as originating from oscillating states of a string source.

This paper follows the line of the second approach. We will consider the fivedimensional solution studied in refs. [13,2], describing a family of non-extremal black holes, which includes the standard five-dimensional Reissner-Nordström solution. In sect. 2 it will be shown that the $\sigma$-model becomes, in the vicinity of the event horizon, an exactly solvable conformal field theory, which is formally related to a particular member of the class of models solved in ref. [14] (or the supersymmetric version given in ref. [15]). In sect. 3 the corresponding (type II) superstring model will be solved and the exact physical spectrum will be determined. This has the expected $T$-duality invariance under the exchange of winding and Kaluza-Klein momentum charges. The simplicity of the horizon theory opens the possibility of having a tractable framework to quantitatively investigate certain types of interactions with infalling matter.

While for extremal black holes the derivation of the statistical entropy reduces to the counting of BPS states (i.e. the counting of all marginal supersymmetric deformations of the conformal $\sigma$-model describing black holes with the same asymptotic charges), for nonextremal black holes it is unclear what states should be counted. The formalism developed here might in principle be of use to derive a statistical entropy, under the basic assumption that the entropy of a non-extremal black hole originates from non-supersymmetric perturbations of the conformal $\sigma$-model (or non-BPS excitations of the string source). In

order to count all states with given energy and charges, one then needs to establish a correspondence between the string energy due to the oscillations and the black hole ADM mass. In sect. 4 we include some remarks on this point, though a complete treatment is 
outside the scope of this paper. An appendix gives further details of the solution to the horizon model.

Let us first describe the extremal black hole recently investigated in [11]. The background is represented by the $D=10$ supersymmetric conformal $\sigma$-model with the following bosonic part [16]

$$
\begin{gathered}
L=\left(G_{\mu \nu}+B_{\mu \nu}\right)(x) \partial X^{\mu} \bar{\partial} X^{\nu}+\mathcal{R} \Phi(x) \\
=F(x) \partial u[\bar{\partial} v+K(x) \bar{\partial} u]+\left(g_{m n}+B_{m n}\right)(x) \partial x^{m} \bar{\partial} x^{n}+\partial y_{a} \bar{\partial} y_{a}+\mathcal{R} \Phi
\end{gathered}
$$

where $u=y+t, v=y-t, x_{m}(m=1,2,3,4)$ are non-compact spatial coordinates, $y_{a}$ $(a=1,2,3,4), y$ are coordinates of the 5 -torus, and $\left(r^{2} \equiv x_{m} x_{m}\right)$

$$
\begin{gathered}
g_{m n}=f(x) \delta_{m n}, \quad H^{m n k}=-\frac{2}{\sqrt{g}} \epsilon^{m n k p} \partial_{p} \phi, \quad e^{2 \phi}=f, \quad e^{2 \Phi}=F f \\
f=1+\frac{P}{r^{2}}, \quad F^{-1}=1+\frac{Q_{1}}{r^{2}}, \quad K=\frac{Q_{2}}{r^{2}} .
\end{gathered}
$$

The ten-dimensional $B_{\mu \nu}$ has both electric $Q_{1}$ and magnetic $P$ charges. $Q_{2}$ corresponds to adding a Kaluza-Klein momentum boost in the $y$ direction $(y \sim y+2 \pi R)$. They are related to integers by $\left(\alpha^{\prime}=1\right)$

$$
a Q_{1}=w R, \quad a Q_{2}=\frac{m}{R}, \quad a \equiv \frac{\pi}{4 G},
$$

where $G \equiv G_{5}$ is the five-dimensional Newton constant (related to the volume $(2 \pi)^{4} V$ of the four-torus $a=1,2,3,4$, and the radius $R$ by $\left.G=\pi g^{2} /(4 R V)\right)$.

Upon compactification along $y_{1}, \ldots, y$ one finds the $D=5$ black hole (Einstein-frame) metric

$$
\begin{gathered}
d s_{5}^{2}=-\lambda^{-2 / 3}(r) d t^{2}+\lambda^{1 / 3}(r)\left(d r^{2}+r^{2} d \Omega_{3}^{2}\right), \\
\lambda=\left(1+\frac{Q_{1}}{r^{2}}\right)\left(1+\frac{Q_{2}}{r^{2}}\right)\left(1+\frac{P}{r^{2}}\right) .
\end{gathered}
$$

In the throat region $r \rightarrow 0$ the theory is just described by a direct product of $S L(2, R)$ $(t, y, r)$ and $\mathrm{SU}(2)$ (with Euler angles associated with the angular coordinates of the threesphere) WZW models with equal levels proportional to $P$ [11]. It will be shown below that this conformal model does not describe the extremal limit of the horizon theory of a non-extremal black hole. 


\section{Conformal model for a non-extremal black hole}

The non-extremal solution is conveniently parametrized in terms of 'boost' parameters [2], $\left\{Q_{1}, Q_{2}, P, E\right\} \rightarrow\left\{\alpha, \gamma, \sigma, r_{0}\right\}$, with the relations

$$
\begin{gathered}
Q_{1}=\frac{r_{0}^{2}}{2} \sinh 2 \alpha, \quad P=\frac{r_{0}^{2}}{2} \sinh 2 \gamma, \quad Q_{2}=\frac{r_{0}^{2}}{2} \sinh 2 \beta, \\
E=\frac{a r_{0}^{2}}{2}(\cosh 2 \alpha+\cosh 2 \gamma+\cosh 2 \beta), \quad a \equiv \frac{\pi}{4 G} .
\end{gathered}
$$

In terms of the $\sigma$-model metric, the solution reads

$$
\begin{gathered}
d s^{2}=\left(1+\frac{r_{0}^{2} \sinh ^{2} \alpha}{r^{2}}\right)^{-1}\left[-d t^{2}+d y^{2}+\frac{r_{0}^{2}}{r^{2}}(\cosh \beta d t+\sinh \beta d y)^{2}\right]+d y_{a}^{2} \\
+\left(1+\frac{r_{0}^{2} \sinh ^{2} \gamma}{r^{2}}\right)\left[\left(1-\frac{r_{0}^{2}}{r^{2}}\right)^{-1} d r^{2}+r^{2} d \Omega_{3}^{2}\right], \\
e^{2 \Phi}=\left(1+\frac{r_{0}^{2} \sinh ^{2} \gamma}{r^{2}}\right)\left(1+\frac{r_{0}^{2} \sinh ^{2} \alpha}{r^{2}}\right)^{-1}, \quad H=2 P \epsilon_{3},
\end{gathered}
$$

where $\epsilon_{3}$ is the volume form on the unit three-sphere. The extremal limit corresponds to the limit $r_{0} \rightarrow 0$ with at least one of the boost parameters $\alpha, \beta, \gamma \rightarrow \pm \infty$ keeping the associated charges (2.1) fixed. For the extremal solution there exists a renormalization scheme in which the corresponding supersymmetric $\sigma$-model (11.1) is conformal to all orders in the $\alpha^{\prime}$ expansion. The non-extremal black hole is not of the chiral null form and is likely to receive $\alpha^{\prime}$ corrections in all renormalization schemes. However, for large black holes $\alpha^{\prime}$ corrections to the metric (2.3) can be neglected in the horizon region. In particular, they would affect terms in the entropy formula which are subleading in the macroscopic limit. Only the leading part of the entropy is expected to be universal (i.e. the same for type II or heterotic embeddings), and given by the Bekenstein-Hawking formula.

The five-dimensional (Einstein-frame) metric arising upon dimensional reduction has the symmetric form:

$$
d s_{5}^{2}=-h^{-2 / 3}\left(1-\frac{r_{0}^{2}}{r^{2}}\right) d t^{2}+h^{1 / 3}\left[\left(1-\frac{r_{0}^{2}}{r^{2}}\right)^{-1} d r^{2}+r^{2} d \Omega_{3}^{2}\right],
$$

where

$$
h=\left(1+\frac{r_{0}^{2} \sinh ^{2} \alpha}{r^{2}}\right)\left(1+\frac{r_{0}^{2} \sinh ^{2} \gamma}{r^{2}}\right)\left(1+\frac{r_{0}^{2} \sinh ^{2} \beta}{r^{2}}\right) .
$$


The background represents a solution of extended $D=5$ supergravity (with $N=1$ supersymmetry in the extremal case). The solution is manifestly invariant under permutations of the three boost parameters as required by $U$-duality (arising as a subgroup of the global $E_{6}$ symmetry of $D=5$ supergravity). The event horizon is at $r=r_{0}$, and $r=0$ represents a regular inner horizon. The entropy and the Hawking temperature are given by

$$
\begin{gathered}
S=\frac{A_{5}}{4 G_{5}}=2 \pi a r_{0}^{3} \cosh \alpha \cosh \beta \cosh \gamma, \\
T=\frac{1}{2 \pi r_{0} \cosh \alpha \cosh \beta \cosh \gamma} .
\end{gathered}
$$

It is convenient to introduce a new coordinate $\hat{r}$ defined by

$$
\hat{r}=\frac{r}{r_{0}}+\sqrt{\frac{r^{2}}{r_{0}^{2}}-1}
$$

The $r, \Omega_{3}$ part of the metric (2.3) takes the form:

$$
\begin{gathered}
d s^{2}=\frac{1}{\hat{r}^{2}}\left(r^{2}+r_{0}^{2} \sinh ^{2} \gamma\right)\left(d \hat{r}^{2}+\hat{r}^{2} d \Omega_{3}^{2}\right)=r_{0}^{2}\left(\cosh ^{2} \rho+\sinh ^{2} \gamma\right)\left(d \rho^{2}+d \Omega_{3}^{2}\right) \\
r=r(\hat{r})=\frac{1}{2} r_{0}\left(\hat{r}+\hat{r}^{-1}\right)=r_{0} \cosh \rho, \quad \rho \equiv \ln \hat{r} \\
d \Omega_{3}^{2}=d \theta^{2}+\sin ^{2} \theta d \varphi^{2}+\cos ^{2} \theta d \psi^{2}
\end{gathered}
$$

The background can then be represented by the $D=10$ supersymmetric conformal $\sigma$ model, with the bosonic part $\left(\hat{r}^{2}=x_{m} x_{m}\right)$ :

$$
\begin{aligned}
L= & F(\hat{r})\left[-\partial \hat{t} \bar{\partial} \hat{t}+\partial \hat{y} \bar{\partial} \hat{y}+\frac{r_{0}^{2}}{r^{2}(\hat{r})} \partial \hat{t} \bar{\partial} \hat{t}\right]+B_{y t}(\partial y \bar{\partial} t-\bar{\partial} y \partial t) \\
& +\left(g_{m n}+B_{m n}\right)(\hat{r}) \partial x^{m} \bar{\partial} x^{n}+\partial y_{a} \bar{\partial} y_{a}+\mathcal{R} \Phi, \\
& \hat{y} \equiv \sinh \beta t+\cosh \beta y, \quad \hat{t} \equiv \cosh \beta t+\sinh \beta y,
\end{aligned}
$$

where the dilaton and $H^{m n k}$ were given in eq. (2.4), $g_{m n}=\hat{f}(x) \delta_{m n}$, and $B_{y t}=Q_{1} F / r^{2}$, with

$$
\hat{f}=\frac{1}{\hat{r}^{2}}\left(r^{2}(\hat{r})+r_{0}^{2} \sinh ^{2} \gamma\right), \quad F^{-1}=1+\frac{r_{0}^{2} \sinh ^{2} \alpha}{r^{2}(\hat{r})} .
$$

This background can be embedded in both type II and heterotic superstring theories, and it can be physically interpreted as representing a bound state of a closed macroscopic string wrapped $w$ times around $y$ (with a momentum boost $m / R$ ), and a solitonic five-brane 
wrapped $P$ times around the five-torus (the special cases $w=0$ or $P=0$ describe the geometry of a macroscopic string or a five-brane).

In the horizon region $\rho \rightarrow 0$ the $\sigma$-model action takes the form

$$
\begin{gathered}
I=\frac{1}{\pi} \int d^{2} \sigma L_{\rho \rightarrow 0}=I_{1}+I_{2}, \\
I_{1}=\frac{1}{\pi \cosh ^{2} \alpha} \int d^{2} \sigma\left(\partial \hat{y} \bar{\partial} \hat{y}-\rho^{2}(\partial \hat{t}+\tanh \alpha \partial \hat{y})(\bar{\partial} \hat{t}-\tanh \alpha \bar{\partial} \hat{y})\right) \\
I_{2}=\frac{1}{\pi} \int d^{2} \sigma\left(\kappa^{2} \partial \rho \bar{\partial} \rho+\partial \theta^{\prime} \bar{\partial} \theta^{\prime}+\partial \varphi^{\prime} \bar{\partial} \varphi^{\prime}+\partial \psi^{\prime} \bar{\partial} \psi^{\prime}\right), \quad \kappa \equiv r_{0} \cosh \gamma
\end{gathered}
$$

where the free internal coordinates and the dilaton term have been omitted. We have also rescaled the angular coordinates so that they have a standard kinetic term. 1 The new coordinates $\theta^{\prime}, \varphi^{\prime}, \psi^{\prime}$ are periodic with period proportional to $\kappa$. For large $\kappa$ their winding numbers can be ignored, so we can treat these coordinates just as free uncompact coordinates. In the extremal limit $\kappa^{2}$ reduces to the magnetic charge $P$. For the ReissnerNordström case, $\alpha=\beta=\gamma, \kappa$ is just the Schwarzschild radius $R_{s}=r_{0} \cosh \alpha$ (see eq. (2.5)). In general, it will depend on the three charges (2.1) and on the ADM energy (2.2).

It is easy to check by direct calculation that the $T$-duality invariance of the original $\sigma$-model (2.13) is preserved in the horizon theory (2.16), i.e. a $T$-duality transformation in the $y$ direction leads to the same $\sigma$-model (2.16) with $\alpha$ and $\beta$ exchanged.

The $\sigma$-model (1.1) describing the extremal black hole solution has a different $(S L(2, R) \times S U(2)$ WZW $)$ structure near the horizon [11]. This cannot be recovered by taking the extremal limit on eq. (2.16). 2 The horizon limit $r \rightarrow r_{0}$ and the extremal limit $r_{0} \rightarrow 0$ do not commute, i.e. the horizon theory of the extremal black hole is in no limit associated with the physics of the non-extremal black hole.

1 For large $\kappa\left(\kappa^{2} \gg \alpha^{\prime}\right)$, the dynamics in the horizon region can be locally described by flat coordinates. Expanding $\theta=\theta_{0}+\frac{\theta^{\prime}}{k}$, one has $\kappa^{2} \partial \theta \partial \theta \rightarrow \partial \theta^{\prime} \partial \theta^{\prime}$, and $\sin ^{2} \theta \cong \sin ^{2} \theta_{0}$, so that $\varphi^{\prime}=\kappa \sin \theta_{0} \varphi, \quad \psi^{\prime}=\kappa \cos \theta_{0} \psi$. We also drop a total derivative term $\partial \varphi^{\prime} \bar{\partial} \psi^{\prime}-\bar{\partial} \varphi^{\prime} \partial \psi^{\prime}$, which is justified in the large- $\kappa$ limit.

2 In the limit $r_{0} \rightarrow 0$, one has $\rho \rightarrow \infty$, so the expansion for small $\rho$ leading to (2.16) does not apply. To take the extremal limit, it is useful to introduce a new coordinate $\tilde{\rho}$ which is regular as $r_{0} \rightarrow 0$, by the shift $\tilde{\rho}=\rho+\ln r_{0} / 2$, so that $r=e^{\rho}+\frac{r_{0}^{2}}{4} e^{-\rho}$. 


\section{Spectrum in the horizon region}

The analysis of the physical spectrum of the model (2.16) is of interest, in particular, to establish a correspondence between the asymptotic parameters characterizing the black hole geometry and the quantum numbers of a string moving in the vicinity of the horizon. Throughout we restrict the discussion to the sector where the winding number and KaluzaKlein momentum of the string state coincide with those of the black hole geometry. We will consider the type II superstring theory embedding of the background discussed in the previous section. To simplify the notation, during part of the derivation the fermionic fields will be omitted. The only substantial difference with the purely bosonic case (apart from straightforward addition of fermion mode oscillators) is a normal ordering constant.

Let us introduce Minkowski world-sheet coordinates, $\sigma_{ \pm}=\tau \pm \sigma$. The non-trivial part of the Lagrangian (2.16) is

$$
\pi L=\partial_{+} z \partial_{-} z-\tilde{\rho}^{2}\left(\partial_{+} T+A \partial_{+} z\right)\left(\partial_{-} T-A \partial_{-} z\right)+\partial_{+} \tilde{\rho} \partial_{-} \tilde{\rho}
$$

where

$$
z \equiv \frac{\hat{y}}{\cosh \alpha}, \quad T \equiv \frac{\hat{t}}{\kappa \cosh \alpha}, \quad \tilde{\rho} \equiv \kappa \rho, \quad A \equiv \frac{\tanh \alpha}{r_{0} \cosh \gamma} .
$$

The boundary conditions for the fields $X^{ \pm} \equiv \tilde{\rho} e^{ \pm T}$ and $z$, as follows from $y \sim y+2 \pi w R$, are given by

$$
\begin{gathered}
X^{ \pm}(\sigma+\pi)=e^{ \pm 2 \pi \varepsilon} X^{ \pm}(\sigma), \quad \varepsilon=\frac{2 \pi w m}{S}=\frac{a r_{0} \sinh \alpha \sinh \beta}{\cosh \gamma}, \\
z(\sigma+\pi)=z(\sigma)+2 \pi w \tilde{R}, \quad \tilde{R}=R \frac{\cosh \beta}{\cosh \alpha},
\end{gathered}
$$

where $S$ is the black hole entropy, eq. (2.7). We will also need to relate the momentum $p_{z}$ to the momentum $p_{y}=m / R=a Q_{2}=a r_{0}^{2} \sinh \beta \cosh \beta$. More generally, consider the canonical momenta $\Pi_{y}, \Pi_{z}, \Pi_{T}, \Pi_{t}$ conjugate to $y, z, T, t$. From eqs. (2.14) and (3.2), we find

$$
\begin{aligned}
& \Pi_{y}=\Pi_{z} \frac{\cosh \beta}{\cosh \alpha}+\Pi_{T} \frac{\sinh \beta}{r_{0} \cosh \gamma \cosh \alpha}, \\
& \Pi_{t}=\Pi_{z} \frac{\sinh \beta}{\cosh \alpha}+\Pi_{T} \frac{\cosh \beta}{r_{0} \cosh \gamma \cosh \alpha},
\end{aligned}
$$

3 Earlier studies in this direction can be found in refs. [3, 17], where the string spectrum in the presence of cone singularities (associated with Euclidean Rindler space at finite temperature) is discussed. 
and the inverse relation

$$
\begin{gathered}
\Pi_{T}=r_{0} \cosh \gamma \cosh \alpha\left(\Pi_{t} \cosh \beta-\Pi_{y} \sinh \beta\right), \\
\Pi_{z}=\cosh \alpha\left(-\Pi_{t} \sinh \beta+\Pi_{y} \cosh \beta\right) .
\end{gathered}
$$

Using eq. (3.5), we obtain

$$
\begin{gathered}
p_{z}=\int_{0}^{\pi} d \sigma \Pi_{z}=\frac{m}{\tilde{R}}-B P_{T}=\frac{m}{\tilde{R}}\left(1-\frac{2 \pi P_{T}}{S}\right) \\
P_{T} \equiv \int_{0}^{\pi} d \sigma \Pi_{T}, \quad B \equiv \frac{\tanh \beta}{r_{0} \cosh \gamma} .
\end{gathered}
$$

$P_{T}$ generates translations in Rindler time.

The structure of the theory (3.1) is that of an interacting theory with non-trivial boundary conditions. Nevertheless, it can be solved exactly. Indeed, the Lagrangian (3.1) has the same form as a world-sheet Lagrangian related to the $(a=\sqrt{3})$ Kaluza-Klein Melvin Universe [15] :

$$
\pi L_{\mathrm{Melvin}}=\partial_{+} y^{\prime} \partial_{-} y^{\prime}+\rho^{2}\left(\partial_{+} \varphi+A \partial_{+} y^{\prime}\right)\left(\partial_{-} \varphi-A \partial_{-} y^{\prime}\right)+\partial_{+} \rho \partial_{-} \rho
$$

where $y^{\prime}$ is a boson compactified on a circle of radius $R^{\prime}$, and $\rho, \varphi$ are polar coordinates. More precisely, the Kaluza-Klein magnetic flux tube Universe is described by the Lagrangian which is $T$-dual to (3.10) in the $y^{\prime}$ direction (with the addition of other free directions). A general class of magnetic flux conformal models interpolating between the dilatonic $(a=1)$ Melvin model and the Kaluza-Klein Melvin model (and their T-duals) were solved in ref. [15], with the spectrum and the modular invariant partition function determined (a larger family was previously treated in [14] for the bosonic string theory).

Instead of repeating the derivation of ref. [15], we will settle the appropriate dictionary between the models (3.1) and (3.10) (the direct solution of the model (3.1) is outlined in the appendix). For this purpose, it is convenient to go to Euclidean Rindler time, $T \rightarrow-i T$, $P_{T} \rightarrow i P_{T}$ (at the end we shall return to the Minkowski-signature Rindler time). The coordinate $\varphi$ can then be identified with $T$ (and $A \rightarrow i A$ ). The operator $P_{T}$ will be identified with the angular momentum operator $\hat{J}=\hat{J}_{R}+\hat{J}_{L}$ of the model (3.10) which generates shifts in $\varphi$.

There are two differences between the theories (3.1) and (3.10) : a) in (3.10), the field $\rho e^{i \varphi}$ is single-valued (being the physical Cartesian uncompact coordinate), whereas in the 
theory (3.1) this field obeys the boundary condition (3.3); b) in the magnetic flux tube model (3.10), the momentum $p_{y^{\prime}}$ is equal to $m^{\prime} / R^{\prime}$, whereas the analogous $p_{z}$ is given by eq. (3.9).

The spectrum of the model (3.10) is not invariant under a $T$-duality transformation in $y^{\prime}$ (the partition function of course it is). In fact, $T$-duality maps to a different $\sigma$-model, the Kaluza-Klein Melvin theory. 1 However, the physical spectrum of the model (3.1) must be invariant under $T$-duality: our original Lagrangian (2.16) is self-dual under a $T$-duality transformation in the $y$-direction (with the change $(\alpha, \beta) \rightarrow(\beta, \alpha)$ ). Indeed, we will show that, by taking into account the two differences (a) and (b) between the models pointed out above, the resulting spectrum will enjoy the expected symmetry.

The Hamiltonian corresponding to the model (3.10) is given by (see eq. (6.8) in ref. [15] with $q=0$ and $\beta$ instead of $A$ )

$$
\begin{gathered}
\hat{H}=\hat{N}_{L}+\hat{N}_{R}-\frac{1}{2} p_{t}^{2}+\frac{1}{2} p_{\alpha}^{2}+\frac{1}{2} p_{y^{\prime}}^{2}+\frac{1}{2}(w R-A \hat{J})^{2}-\hat{\gamma}\left(\hat{J}_{R}-\hat{J}_{L}\right) \\
\hat{\gamma}=A p_{y^{\prime}}, \quad \hat{J}=\hat{J}_{R}+\hat{J}_{L}
\end{gathered}
$$

where the index $\alpha$ stands for extra free coordinates. 5 The operators $\hat{N}_{R, L}=N_{R, L}-a$ contain the free superstring theory Regge intercepts $a^{(\mathrm{R})}=0, a^{(\mathrm{NS})}=1 / 2$, e.g. in the Ramond sector,

$$
\begin{gathered}
N_{R}^{(\mathrm{R})}=\sum_{n=1}^{\infty} n\left(b_{n+}^{\dagger} b_{n+}+b_{n-}^{\dagger} b_{n-}+b_{n \alpha}^{\dagger} b_{n \alpha}+d_{n}^{*} d_{n}+d_{-n} d_{-n}^{*}+d_{-n \alpha} d_{n \alpha}\right) \\
{\left[b_{n}, b_{k}^{\dagger}\right]=\delta_{n k}=\left\{d_{n}, d_{k}^{*}\right\} .}
\end{gathered}
$$

The free creation and annihilation operators $b_{n}, b_{n}^{\dagger}$ and $d_{n}, d_{n}^{*}$ are associated with the bosonic degrees of freedoms represented by $\rho e^{ \pm i \varphi}$ and the fermionic superpartners. Here the light-cone gauge has been used to remove oscillator modes corresponding to the degrees

4 This follows from the standard T-duality rules; the Kaluza-Klein Melvin model has a vector gauge field, which under $T$-duality goes into the axial gauge field of (3.10). Instead, the $(a=1)$ dilatonic Melvin theory has a purely left gauge coupling; the model is self-dual, with a $T$-duality invariant spectrum [15].

5 For simplicity, we shall consider only states with zero winding number and momentum in the internal coordinates $a=1,2,3,4$, so that $p_{\alpha}^{2}$ only contains contributions from the variables $\theta^{\prime}, \varphi^{\prime}, \psi^{\prime}$. 
of freedom associated with $y^{\prime}$ and $t$ (the corresponding term $-\partial_{+} t \partial_{-} t$ was omitted in eq. (3.10) since $t$ is decoupled). In the Ramond sector, the operator $\hat{J}_{R}$ is given by

$$
\begin{gathered}
\hat{J}_{R}=-b_{0}^{\dagger} b_{0}-\frac{1}{2}+\sum_{n=1}^{\infty}\left(b_{n+}^{\dagger} b_{n+}-b_{n-}^{\dagger} b_{n-}\right)+\hat{K}_{R}=P_{R}, \\
\hat{K}_{R}^{(\mathrm{R})}=-\left[d_{0}^{*}, d_{0}\right]+\sum_{n=1}^{\infty}\left(d_{n}^{*} d_{n}+d_{-n} d_{-n}^{*}\right) .
\end{gathered}
$$

The expression of $\hat{J}_{L}=P_{L}$ is similar, with a reversal of the sign of the orbital angular momentum terms.

The analogue of $\hat{\gamma}$ for the model (3.1) is $\hat{\gamma}=i A p_{z}$. However, because of the twisted boundary condition (3.3) in $X^{ \pm}$, the parameter $\hat{\gamma}$ will be shifted by $i \varepsilon$, so it must be replaced by

$$
\hat{\gamma} \longrightarrow \tilde{\gamma}=\hat{\gamma}+i \varepsilon=\frac{4 \pi i m w}{S}+A B P_{T}
$$

The case $m w=0$, implying $\tilde{\gamma}=0$, is special and it will be discussed separately.

Taking into account these changes, we can now write down the Virasoro conditions, determining the physical spectrum of the Euclidean horizon theory:6

$$
\begin{gathered}
\hat{H}_{\mathrm{Eucl}}=\hat{N}_{L}+\hat{N}_{R}+\frac{1}{2} p_{\alpha}^{2}+\frac{1}{2}\left(\frac{m}{\tilde{R}}-i B P_{T}\right)^{2}+\frac{1}{2}\left(w \tilde{R}-i A P_{T}\right)^{2} \\
-\left(\frac{4 \pi i m w}{S}+A B P_{T}\right)\left(P_{R}-P_{L}\right)=0, \quad P_{T}=P_{R}+P_{L} \\
\hat{N}_{R}-\hat{N}_{L}=m w
\end{gathered}
$$

where $A$ and $B$ have been defined in eqs. (3.2) and (3.9).

Let us now formally return to Minkowski-signature Rindler time by $P_{R, L} \rightarrow-i P_{R, L}$. We obtain

$$
\begin{gathered}
\hat{H}=\hat{L}_{0}+\hat{\tilde{L}}_{0}=\hat{N}_{L}+\hat{N}_{R}+\frac{1}{2} p_{\alpha}^{2}+\frac{1}{2}\left(\frac{m}{\tilde{R}}-B P_{T}\right)^{2}+\frac{1}{2}\left(w \tilde{R}-A P_{T}\right)^{2} \\
+A B\left(P_{R}^{2}-P_{L}^{2}\right)-\frac{4 \pi w m}{S}\left(P_{R}-P_{L}\right)=0 \\
\hat{L}_{0}-\hat{\tilde{L}}_{0}=\hat{N}_{R}-\hat{N}_{L}-m w=0
\end{gathered}
$$

\footnotetext{
${ }^{6}$ In the bosonic theory the Hamiltonian has an extra normal-ordering contribution $\frac{1}{2} \tilde{\gamma}^{2}$.
} 
Using the definition of $A, B$ and the relation between the $\alpha, \beta$ and $m, w$ (se eqs. (1.4), (2.1)), eq. (3.18) can be written in the form

$$
\begin{gathered}
2 \hat{H}=2 \hat{N}_{L}+2 \hat{N}_{R}+p_{\alpha}^{2}+\left(\frac{m^{2}}{\tilde{R}^{2}}+w^{2} \tilde{R}^{2}\right)\left(1-\frac{2 \pi P_{T}}{S}\right)^{2} \\
-\frac{8 \pi m w}{S^{2}}\left(P_{R}-P_{L}\right)\left(S-\pi P_{T}\right)=0
\end{gathered}
$$

The spectrum is thus invariant under the duality transformation $\alpha \leftrightarrow \beta$ (or $m \leftrightarrow w$, $R \leftrightarrow 1 / R)$

In contrast with the Euclidean theory, now the operators $P_{L}, P_{R}$ are no longer angular momentum operators. They are defined by

$$
P_{L, R}=\int_{0}^{\pi} d \sigma \Pi_{L, R}, \quad \Pi_{L, R}=\frac{\tilde{\rho}^{2}}{2 \pi}\left(\partial_{ \pm} T \pm A \partial_{ \pm} z\right), \quad P_{T}=P_{R}+P_{L}
$$

In terms of oscillator modes, they can still be formally written as in eq. (3.14) with a factor $-i$ in front, but it is convenient to return to the conventional notation $\alpha_{n}^{\mu}$. This is done by defining

$$
b_{n \pm} \equiv \frac{\alpha_{n}^{1} \pm \alpha_{n}^{0}}{\sqrt{2 n}}, \quad b_{n \pm}^{\dagger} \equiv \frac{\alpha_{-n}^{1} \mp \alpha_{-n}^{0}}{\sqrt{2 n}}, \quad n=1,2, \ldots
$$

and similarly for the left modes $\tilde{\alpha}_{n}^{\mu}$. The correct conjugation properties of the $b_{n}, b_{n}^{\dagger}$ operators follow from $\left(\alpha_{-n}^{\mu}\right)^{\dagger}=\alpha_{n}^{\mu}$ (see appendix). The resulting expressions for $\hat{N}_{R}$ and $P_{R}$ contain the familiar contributions $-\alpha_{-n}^{0} \alpha_{n}^{0}+\alpha_{-n}^{1} \alpha_{n}^{1}$ and $\frac{i}{n}\left(\alpha_{-n}^{1} \alpha_{n}^{0}-\alpha_{-n}^{0} \alpha_{n}^{1}\right)$. The way the time enters into the Lagrangian of the model (3.1) is different from the analogue model (3.10). This makes a difference in the choice of the light-cone gauge (for the Euclidean theory it makes of course no difference). In order to avoid negative-norm states in the spectrum, now the light-cone gauge must be used to remove the oscillators $\alpha_{n}^{0}, \alpha_{n}^{1}, n \neq 0$. As a result, only a constant zero mode contribution $x^{ \pm}, \tilde{x}^{ \pm}$(related to $b_{0}, b_{0}^{\dagger}$ by $\left.b_{0}, b_{0}^{\dagger}=\sqrt{-i} x^{\mp}, \tilde{b}_{0}, \tilde{b}_{0}^{\dagger}=\sqrt{i} \tilde{x}^{\mp}\right)$ remains in the Rindler operators $P_{R, L}$. Now the different operators take the form $(m w \neq 0)$

$$
\begin{gathered}
P_{R}=x^{+} x^{-}, \quad P_{L}=\tilde{x}^{+} \tilde{x}^{-}, \quad\left[x^{-}, x^{+}\right]=i=\left[\tilde{x}^{+}, \tilde{x}^{-}\right] \\
N_{R}^{(\mathrm{R})}=\sum_{n=1}^{\infty}\left[\alpha_{-n z} \alpha_{n z}+\alpha_{-n \alpha} \alpha_{n \alpha}+n\left(d_{-n z} d_{n z}+d_{-n \alpha} d_{n \alpha}\right)\right], \\
{\left[\alpha_{n}^{\mu}, \alpha_{k}^{\nu}\right]=n \delta_{n+k} \eta^{\mu \nu}, \quad\left\{d_{n}, d_{k}\right\}=\delta_{n+k},}
\end{gathered}
$$


where the modes with subindex $z$ are those associated with the field $z$ and its superpartners. The physical space is simply constructed by applying the free creation operators on the vacuum Fock state. The full treatment of the zero mode sector $x^{ \pm}, \tilde{x}^{ \pm}$involves long technical details, which we plan to present elsewhere. 0 The calculation given here will not depend on the explicit form of $P_{L}, P_{R}$.

In the special case $m w=0=\tilde{\gamma}$, the fields $X^{ \pm}$are single-valued. As a result, translational invariance of the Rindler Cartesian plane $X^{1}=\tilde{\rho} \cosh T, X^{0}=\tilde{\rho} \sinh T$ is restored. The zero-mode operators $x^{ \pm}$and $\tilde{x}^{ \pm}$, are traded by center-of-mass coordinates and momentum $x_{0}, x_{1}$ and $p_{0}, p_{1}$. The bosonic zero mode parts of $P_{L}, P_{R}$ are replaced by $\frac{1}{2}\left(x_{0} p_{1}-x_{1} p_{0}\right)$, and the Hamiltonian reads $(w=0)$

$$
\hat{H}=\hat{N}_{R}+\hat{N}_{L}+\frac{1}{2} p_{\alpha}^{2}+\frac{1}{2} p_{1}^{2}-\frac{1}{2} p_{0}^{2}+\frac{m^{2}}{2 \tilde{R}^{2}}\left(1-\frac{2 \pi P_{T}}{S}\right)^{2}
$$

In particular, for a Schwarzschild black hole one simply has

$$
M_{\text {Rind }}^{2} \equiv p_{0}^{2}-p_{1}^{2}-p_{\alpha}^{2}=4 \hat{N}_{L}
$$

$M_{\text {Rind }}^{2}$ represents the invariant mass in the Rindler Cartesian plane. For a black hole with $m w \neq 0, p_{0}, p_{1}$ are not conserved quantum numbers.

In the extremal limit $\alpha, \beta, \gamma \rightarrow \infty, r_{0} \rightarrow 0$, with the charges (2.1) fixed, one obtains

$$
A_{\mathrm{ext}}=B_{\mathrm{ext}}=\frac{1}{\sqrt{P}}, \quad \tilde{\gamma}_{\mathrm{ext}}=\frac{P_{T}}{P}, \quad \frac{m}{\tilde{R}}=w \tilde{R}
$$

where $P$ is the magnetic charge introduced earlier. The Hamiltonian (3.18) becomes

$$
\begin{gathered}
\hat{H}_{\mathrm{ext}}=\hat{N}_{R}+\hat{N}_{L}+\frac{1}{2} p_{\alpha}^{2}+\frac{1}{P}\left[\left(\frac{S}{2 \pi}-P_{T}\right)^{2}-\left(P_{R}-P_{L}\right)\left(\frac{S}{\pi}-P_{T}\right)\right], \\
S=2 \pi \sqrt{m w P} .
\end{gathered}
$$

The Hamiltonian contains a free part and a term multiplied by an overall factor $1 / P$, which involves only the product of the three charges, i.e. it depends only on the entropy and the Rindler energy operator.

7 A system with a similar zero mode structure is discussed in ref. [18]. 


\section{The string source}

\subsection{Rindler energy}

For a classical black hole geometry, the energies measured by a Minkowskian distant observer (the ADM energy) and by an inertial observer moving in the neighborhoods of the horizon (where the $r, t$ part of the metric describes a two-dimensional Rindler space-time) are related by

$$
d E_{\mathrm{R}}=\frac{d E}{2 \pi T_{H}} .
$$

Using the first law of black hole thermodynamics, $d E=T_{H} d S$, we can express the Rindler energy in terms of the entropy:

$$
E_{\mathrm{R}}=\frac{S(E)}{2 \pi}=\frac{A}{8 \pi G} .
$$

Consider for example the Reissner-Nördstrom black hole. From eq. (4.1) we have

$$
d E_{\mathrm{R}}=\frac{r_{+}^{3}}{r_{+}^{2}-r_{-}^{2}} d E, \quad r_{+}^{2}=\mu+\sqrt{\mu^{2}-Q^{2}}, \quad \mu=\frac{4 G E}{3 \pi} .
$$

Integrating this relation we obtain

$$
E_{\mathrm{R}}=\frac{\pi}{4 G} r_{+}^{3} .
$$

In particular, for a neutral black hole, this gives

$$
E_{\mathrm{R}}=\frac{2}{3} E^{3 / 2}\left(\frac{8 G}{3 \pi}\right)^{1 / 2} .
$$

This agrees with a derivation given in ref. [12].

\subsection{Rindler energy of states with $N_{L}=P_{L}=0$}

Equation (4.2) can be explicitly checked for the black hole (2.5) by direct integration. One obtains a relation of the form, $2 \pi E_{\mathrm{R}}=S(E, m, w, P)$, which implicitly determines the ADM energy $E$ in terms of the Rindler energy and the charges, i.e. $E=f\left(E_{R}, m, w, P\right)$.

Let us now return to the physical spectrum of the conformal model. Equation (3.20) can be viewed as determining the eigenvalues of the Rindler operator $P_{T}$ in terms of the charges and the oscillator state characterized by $\hat{N}_{L}, \hat{N}_{R}$ and $P_{R}-P_{L}$. The Rindler operator generates translations in the Rindler time $T$, and thus its eigenvalues determine the Rindler energy of the configuration. 
Consider the particular case of black holes with zero magnetic charge. Let us calculate the Rindler energy of a state with $\hat{N}_{L}=0, P_{L}=0$. We will assume that $\hat{N}_{R}=m w \gg 1$, so that the Regge intercepts can be ignored, and consider the center-of-mass frame where $p_{\alpha}^{2}=0$. The Virasoro conditions (3.19), (3.20) imply

$$
\left(\frac{m}{\tilde{R}}+w \tilde{R}\right)^{2}\left(1-\frac{2 \pi P_{T}}{S}\right)^{2}=0,
$$

or

$$
P_{T}=\frac{S}{2 \pi} .
$$

Strikingly, this is the relation (4.2). Since these states carry the same charges $m, w$ of the $(P=0)$ black hole, this means that the associated ADM mass is the black hole ADM mass! Thus, for a black hole with zero magnetic charge, an oscillating string in the horizon in a $N_{L}=P_{L}=0$ state can be interpreted as a source of the gravitational field: it has the same charges and ADM mass of the black hole geometry.

\subsection{String sources for the non-extremal black hole with zero magnetic charge}

We have seen that the ADM mass eigenvalue associated with states with only rightmoving mode content coincides with the ADM mass of the electrically charged black hole. Let us now find the general states whose mass equals that of the $P=0$ black hole geometry. To address this question, we return to the spectrum (3.20) and look for states where the eigenvalue of the Rindler energy operator is $P_{T}=S / 2 \pi$. For these states, there is a remarkable simplification in the Hamiltonian (3.20), which takes the form

$$
\hat{H}=\hat{N}_{L}+\hat{N}_{R}-\frac{2 \pi w m}{S}\left(P_{R}-P_{L}\right) .
$$

Using eq. (3.19), and $S=2 \pi\left(P_{L}+P_{R}\right)$, we obtain

$$
P_{R}=\frac{S}{2 \pi w m} N_{R}, \quad P_{L}=-\frac{S}{2 \pi w m} N_{L} .
$$

All states in the physical spectrum satisfying (4.9) will have ADM mass eigenvalue coinciding with the ADM mass parameter of the black hole configuration, and thus they can be used as sources. Note that the derivation leading to (4.9) is not valid when $m w=0$, which includes the Schwarzschild geometry. As pointed out above, in this case the zero-mode structure changes, and there is an additional term in the Hamiltonian of the form $-p_{0}^{2}+p_{1}^{2}$. 
A natural question is whether the logarithm of the total number $\Omega(E)$ of states with ADM energy $E$ is related to the Bekenstein-Hawking formula. The condition (4.9) fixes the expectation value of the operators $P_{R, L}$ in terms of $N_{R, L}$. Ignoring power-like factors, the number of states obeying (4.9) is asymptotically given by

$$
\Omega(E)=\sum_{N_{R}=m w}^{\infty} \exp \left[2 a_{R} \sqrt{N_{R}}+2 a_{L} \sqrt{N_{R}-m w}\right],
$$

where for the type II superstring theory, $a_{R, L}=\sqrt{2} \pi$. The sum over $N_{R}$ clearly diverges. It is likely that only a subset of the states obeying (4.9) can constitute the microstates of the black hole. This is the case for the limiting situation of an extremal black hole, which is $N=1$ supersymmetric so it can only be identified with states with $N_{L}=0$. This fixes $N_{R}=m w$, so only one term in the sum (4.10) remains. The number of BPS states with the same asymptotic parameters of the black hole is $\Omega(E) \sim \exp [$ const. $\sqrt{m w}]$. However, the extremal black hole with $P=0$ is not suitable for a comparison with the BekensteinHawking prediction. As discussed in ref. [5], in this case $S \rightarrow 0$, the event horizon becomes singular, and the semiclassical calculation leading to the Bekenstein-Hawking formula does not apply. It would be interesting to understand if for the non-extremal black hole there is an extra condition restricting the set of states that contribute to the sum (4.10), which in the extremal limit reduces to $N_{L}=0$.

To summarize, the horizon region of a class of non-extremal black holes, which includes the standard five-dimensional Reissner-Nördstrom solution, is described by a solvable conformal field theory, and exact formulas for the physical spectrum can be written. There is an ADM mass that can be associated with each state of the spectrum (3.20). This is done by means of the eigenvalue of the Rindler energy operator $P_{T}$ on the state, using the standard relation between Rindler energy and ADM mass. We have seen that states with Rindler energy equal to the black hole entropy over $2 \pi$ are special; in particular, the Hamiltonian in this sector simplifies. To address counting problems, there are some points in the interpretation of the spectrum that need to be clarified. A thorough study is desirable to understand to what extent these states can be regarded as microstates constituting the non-extremal black hole.

Acknowledgements: The author is grateful to A. Sen for a useful discussion. He also wishes to thank G. Horowitz and A. Tseytlin for comments. 


\section{Appendix A. Direct solution of the horizon model}

The general solution to the equations of motion corresponding to the Lagrangian (3.1) is given in terms of three free fields $Z, Y^{+}, Y^{-}$, as follows:

$$
\begin{gathered}
z=Z-A \tilde{\varphi}, \quad T=A\left(Z_{R}-Z_{L}\right)+\frac{1}{2} \ln \frac{Y^{+}}{Y^{-}}, \quad \tilde{\rho}^{2}=Y^{+} Y^{-}, \\
Z=Z_{R}\left(\sigma_{-}\right)+Z_{L}\left(\sigma_{+}\right), \quad Y^{ \pm}=Y_{R}^{ \pm}\left(\sigma_{-}\right)+Y_{L}^{ \pm}\left(\sigma_{+}\right), \\
\partial_{ \pm} \tilde{\varphi}= \pm \frac{1}{2}\left(Y^{-} \partial_{ \pm} Y^{+}-Y^{+} \partial_{ \pm} Y^{-}\right)
\end{gathered}
$$

so that

$$
X^{ \pm}=\tilde{\rho} \exp \left[ \pm A\left(Z_{R}-Z_{L}\right)\right] Y^{ \pm}
$$

We recall that superscripts + and - represent light-cone Lorentz indices, which are not in association with $\sigma_{ \pm}$. The boundary conditions (3.3) and (3.4) are satisfied by (throughout we assume $m w \neq 0)$ :

$$
\begin{gathered}
Y_{R}^{ \pm}=e^{\mp 2 \tilde{\gamma} \sigma_{-}} \chi_{R}^{ \pm}, \quad Y_{L}^{ \pm}=e^{ \pm 2 \tilde{\gamma} \sigma_{+}} \chi_{L}^{ \pm}, \\
\tilde{\gamma}=\varepsilon+A p_{z}, \quad \chi_{R, L}^{ \pm}(\sigma+\pi)=\chi_{R, L}^{ \pm}(\sigma), \\
Z_{L, R}=\sigma_{ \pm} p_{ \pm}+\hat{Z}_{L, R}, \quad \hat{Z}_{L, R}(\sigma+\pi)=\hat{Z}_{L, R}(\sigma) \\
p_{ \pm}= \pm\left(w \tilde{R}-A P_{T}\right)+p_{z}, \quad P_{T}=P_{R}+P_{L}, \\
P_{R, L}=\frac{1}{4 \pi} \int_{0}^{\pi} d \sigma\left(Y_{R, L}^{-} \partial_{\mp} Y_{R, L}^{+}-Y_{R, L}^{+} \partial_{\mp} Y_{R, L}^{-}\right) .
\end{gathered}
$$

The Fourier expansions $\chi_{R, L}^{ \pm}$and $\hat{Z}_{R, L}$ are given by

$$
\begin{array}{ll}
\chi_{R}^{ \pm}=\frac{x^{ \pm}}{\sqrt{\tilde{\gamma}}}+\frac{i}{\sqrt{2}} \sum_{n \neq 0} x_{n}^{ \pm} e^{-2 i n \sigma_{-}}, & \chi_{L}^{ \pm}=\frac{\tilde{x}^{ \pm}}{\sqrt{\tilde{\gamma}}}+\frac{i}{\sqrt{2}} \sum_{n \neq 0} \tilde{x}_{n}^{ \pm} e^{-2 i n \sigma_{+}} \\
\hat{Z}_{R}=z_{0}+\frac{i}{\sqrt{2}} \sum_{n \neq 0} \frac{\alpha_{n z}}{n} e^{-2 i n \sigma_{-}}, & Z_{L}=\tilde{z}_{0}+\frac{i}{\sqrt{2}} \sum_{n \neq 0} \frac{\tilde{\alpha}_{n z}}{n} e^{-2 i n \sigma_{+}} .
\end{array}
$$

Starting from the expression for the classical stress-energy tensor of the theory (3.1) and evaluating it on the general solution (A.1), one finds that it takes the simple form:

$$
T_{ \pm \pm}=\partial_{ \pm} Z_{L, R} \partial_{ \pm} Z_{L, R}+\partial_{ \pm} Y^{+} \partial_{ \pm} Y^{-}
$$

The canonical commutation relations for $X^{ \pm}$imply

$$
\left[x_{n}^{+}, x_{k}^{-}\right]=2(n+i \tilde{\gamma})^{-1} \delta_{n+k}, \quad\left[\tilde{x}_{n}^{+}, \tilde{x}_{k}^{-}\right]=2(n-i \tilde{\gamma})^{-1} \delta_{n+k}
$$




$$
\left[x^{-}, x^{+}\right]=i=\left[\tilde{x}^{+}, \tilde{x}^{-}\right]
$$

In the Euclidean theory the " $i$ " is absent from eq. (A.9), and one can introduce the creation and annihilation operators $b_{n \pm}, b_{n \pm}^{\dagger}$ of eqs. (3.13), (3.14) by rescaling the modes by factors $(n \pm \tilde{\gamma})^{1 / 2}$. In the Minkowskian theory one may formally define $b_{n \pm}, b_{n \pm}^{\dagger}$ operators by rescaling by factors $(n \pm i \tilde{\gamma})^{1 / 2}$, but the $b_{n}^{\dagger}$ are no longer the Hermitian conjugate of the $b_{n}$; the notation becomes misleading, so it is convenient to replace them by the standard $\alpha_{n}^{\mu}$ operators as in eq. (3.22).

The light-cone gauge can be fixed by setting to zero the oscillator part of $\chi_{R, L}^{+}$; as usual, the oscillators of $\chi_{R, L}^{-}$are then determined in terms of transverse oscillators by imposing the Virasoro constraints $L_{n}=\tilde{L}_{n}=0, n \neq 0$. After a bit of algebra one obtains the quantum Virasoro operators, $\hat{L}_{0}=\frac{1}{4 \pi} \int_{0}^{\pi} d \sigma T_{--}, \hat{\tilde{L}}_{0}=\frac{1}{4 \pi} \int_{0}^{\pi} d \sigma T_{++}$, as given by eqs. (3.18), (3.19), with the $P_{R, L}$ and $N_{R}^{(R)}$ of eqs. (3.23), (3.24), and similar expressions for $N_{L}^{(R)}$. In the NS sector the expressions are also similar, with the usual changes that take place in free superstring theory. 


\section{References}

[1] A. Strominger and C. Vafa, hep-th/9601029.

[2] G. Horowitz, J.M. Maldacena and A. Strominger, hep-th/9603109.

[3] L. Susskind, hep-th/9309145.

[4] J.G. Russo and L. Susskind, Nucl. Phys. B437 (1995) 611.

[5] A. Sen, Mod. Phys. Lett. A10 (1995) 2081.

[6] A.W. Peet, Nucl. Phys. B456 (1995) 732.

[7] C.G. Callan, J.M. Maldacena and A.W. Peet, PUPT-1565, hep-th/9510134.

[8] A. Dabholkar, J. Gauntlett, J. Harvey and D. Waldram, hep-th/9511053.

[9] F. Larsen and F. Wilczek, hep-th/9511064.

[10] M. Cvetič and A.A. Tseytlin, Phys. Rev. D53 (1996) 5619; A.A. Tseytlin, Mod. Phys. Lett. A11 (1996) 689.

[11] A.A. Tseytlin, hep-th/9605091.

[12] E. Halyo, A. Rajaraman and L. Susskind, hep-th/9605112.

[13] M. Cvetič and D. Youm, hep-th/9603100.

[14] J.G. Russo and A.A. Tseytlin, Nucl. Phys. B449 (1995) 91.

[15] J.G. Russo and A.A. Tseytlin, Nucl. Phys. B461 (1996) 131.

[16] G.T. Horowitz and A.A. Tseytlin, Phys. Rev. D51 (1995) 2896.

[17] A. Dabholkar, Nucl. Phys. B439 (1995) 650; D.A. Lowe and A. Strominger, Phys. Rev. D51 (1995) 1793.

[18] K. Schoutens, H. Verlinde and E. Verlinde, Phys. Rev. D48 (1993) 2670. 